\title{
Energy distribution of relativistic electrons in the kiloparsec scale jet of M 87 with Chandra
}

\author{
Xiao-Na Sun ${ }^{1}$, Rui-Zhi Yang ${ }^{1}$, Frank M. Rieger ${ }^{1,2}$, Ruo-Yu Liu ${ }^{1}$, and Felix Aharonian ${ }^{1,3,4,5}$ \\ ${ }^{1}$ Max-Planck-Institut für Kernphysik, P.O. Box 103980, 69029 Heidelberg, Germany \\ e-mail: sun@mpi-hd.mpg.de \\ ${ }^{2} \mathrm{ZAH}$, Institut für Theoretische Astrophysik, Heidelberg University, Philosophenweg 12, 69120 Heidelberg, Germany \\ ${ }^{3}$ Dublin Institute for Advanced Studies, 31 Fitzwilliam Place, Dublin 2, Ireland \\ ${ }^{4}$ Gran Sasso Science Institute, 7 viale Francesco Crispi, 67100 L'Aquila (AQ), Italy \\ ${ }^{5}$ MEPHI, Kashirskoe shosse 31, 115409 Moscow, Russia
}

Received 4 August 2017 / Accepted 24 January 2018

\begin{abstract}
The X-ray emission from the jets in active galactic nuclei (AGN) carries important information on the distributions of relativistic electrons and magnetic fields on large scales. We reanalysed archival Chandra observations on the jet of M 87 from 2000 to 2016 with a total exposure of 1460 kiloseconds to explore the X-ray emission characteristics along the jet. We investigated the variability behaviours of the nucleus and the inner jet component HST-1, and confirm indications for day-scale X-ray variability in the nucleus contemporaneous to the 2010 high TeV $\gamma$-ray state. HST-1 shows a general decline in X-ray flux over the last few years consistent with its synchrotron interpretation. We extracted the X-ray spectra for the nucleus and all knots in the jet, showing that they are compatible with a single power law within the X-ray band. There are indications that the resultant X-ray photon index exhibit a trend, with slight but significant index variations ranging from $\simeq 2.2$ (e.g. in knot D) to $\simeq 2.4-2.6$ (in the outer knots $\mathrm{F}, \mathrm{A}$, and $\mathrm{B}$ ). When viewed in a multiwavelength context, a more complex situation can be seen. Fitting the radio to X-ray spectral energy distributions (SEDs) assuming a synchrotron origin, we show that a broken power-law electron spectrum with break energy $E_{\mathrm{b}}$ around $1(300 \mu G / B)^{1 / 2}$ $\mathrm{TeV}$ allows a satisfactory description of the multiband SEDs for most of the knots. However, in the case of knots B, C, and D we find indications that an additional high-energy component is needed to adequately reproduce the broad-band SEDs. We discuss the implications and suggest that a stratified jet model may account for the differences.
\end{abstract}

Key words. X-rays: galaxies - galaxies: individual: M 87 - radiation mechanisms: non-thermal

\section{Introduction}

M 87 (Virgo A, NGC 4486, 3C 274), the giant elliptical galaxy located in the Virgo cluster at a distance of $16.7 \pm 0.6 \mathrm{Mpc}$ $\left(1^{\prime \prime}=78 \mathrm{pc}\right)$, is one of the closest radio galaxies (Blakeslee et al. 2009; Jordán et al. 2005). It is known to host a central black hole with a mass of $\simeq(3-6) \times 10^{9} M_{\odot}$ (Macchetto et al. 1997; Gebhardt et al. 2011; Walsh et al. 2013) and a one-sided 30" scale jet (Marshall et al. 2002; Harris et al. 2003). The jet is characterised by a viewing angle between $10^{\circ}-25^{\circ}$ and reveals superluminal motion of optical components of (4-6)c within $6^{\prime \prime}$ of the nucleus based on HST observations (Biretta et al. 1999). Its jet power $P_{\mathrm{j}}$ is somewhat uncertain with estimates ranging from a few times $10^{43} \mathrm{erg} \mathrm{s}^{-1}$ up to $10^{45} \mathrm{erg} \mathrm{s}^{-1}$ (e.g. Reynolds et al. 1996; de Gasperin et al. 2012; Mościbrodzka et al. 2016; Levinson \& Globus 2017). Owing to its proximity and high surface brightness at radio wavelengths and above, M 87 has become a key laboratory to investigate the property of relativistic jets (e.g. Doeleman et al. 2012; Hada et al. 2016; Mertens et al. 2016; Britzen et al. 2017).

Both the nucleus and several bright jet knots have been detected at radio, optical, and X-ray wavelengths. Their broadband spectral energy distributions (SEDs) have been extensively studied (e.g. Biretta et al. 1991; Sparks et al. 1996; Perlman et al. 2001; Zhang et al. 2010). The high-resolution observations performed by Chandra in the X-ray band make it possible to investigate the spectral energy distributions of the jet substructures in or close to the spectral cut-off regions, imposing important constraints on radiation models (e.g. Marshall et al. 2002; Wilson \& Yang 2002; Perlman \& Wilson 2005; Waters \& Zepf 2005; Zhang et al. 2010).

Owing to the accumulative exposure and the recently enhanced software tools of Chandra, an improved analysis can now be performed to derive more accurate spectrometric information and to further investigate radiation mechanisms in this region. In this paper we present the results of a detailed analysis of the Chandra data on the M 87 nucleus and knots observed from 2000 to 2016. The paper is structured as follows. In Sect. 2 we describe details of the Chandra data reduction process, light curve analysis, and spectral analysis. In Sect. 3 we construct the radio to X-ray SEDs of the substructures (knots) in M 87 and provide model fits to infer the spectral distributions of the parent particles. We discuss the consequences in Sect. 4.

\section{Chandra data analysis}

The Chandra X-ray Observatory (CXO), launched in 1999, provides unprecedented angular resolution $\leq 0.5^{\prime \prime} \mathrm{X}$-ray imaging and spectroscopy over the energy band $0.1-10 \mathrm{keV}(15-0.12 \mathrm{~nm})$ (Weisskopf et al. 2000, 2002). Its Science Instrument Module (SIM) holds the two focal plane instruments, the Advanced CCD Imaging Spectrometer (ACIS) and the High Resolution Camera (HRC). The ACIS is used for spectroscopic studies in the 


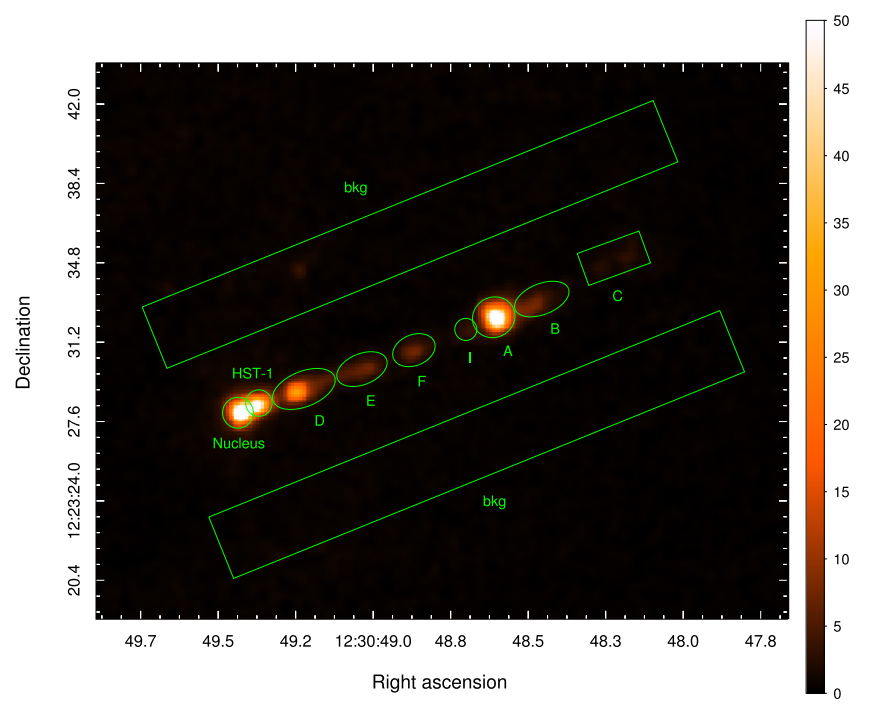

Fig. 1. Image from the observation on July 30, 2000 (ObsID 1808), in the $0.2-10 \mathrm{keV}$, binned in $0 . ' 123$ per pixel and smoothed with a Gaussian of $F W H M=0 ! 3$. The source and background regions are indicated by green shapes. Units of right ascension are hours:minutes:seconds, and units of declination are degrees:arcminutes:arcseconds.

energy range $0.2-10 \mathrm{keV}$. The HRC accurately records the position, number, and energy of X-rays, and images over the range of $0.1-10 \mathrm{keV}^{1}$. In this paper, the Chandra data reduction and spectra extraction are performed using CIAO (v4.8) tool and the Chandra Calibration Database (CALDB, v4.7.2). We perform the spectral analysis with Sherpa ${ }^{2}$.

\subsection{Data preparation}

We collected the Chandra ACIS timed exposure (TE) mode observations from April 15, 2000 (ObsID 517), to June 12, 2016 (ObsID 18856), to perform a detailed analysis of the M 87 jet and nucleus. In Table A.1 we list all the observations, including 99 observations with a $0.4 \mathrm{~s}$ frame time and 13 observations with a 3.2/3.1 s frame time. The total exposure time is over 1460 kiloseconds. Figure 1 provides an exemplary case, showing the ObsID $1808 \mathrm{X}$-ray image binned into 0.' 123 per pixel and smoothed with a Gaussian of $F W H M=0$. . 3 .

To avoid pile-up (i.e. two or more photons arriving at the same pixel during a single frame time, thus mimicking a single event with the sum of the energies; see e.g. Davis 2001), we generally selected the observations with frame time of $0.4 \mathrm{~s}$ for all substructures analyses. However, because knots E, F, I, B, and $\mathrm{C}$, as shown in Fig. 1, are much fainter than the nucleus, HST1 , knot D, and knot A, we also included the observations with frame time of 3.2/3.1 s in the analysis of these knots.

To reduce uncertainties caused by the position offsets of different observations, we performed the astrometric corrections as follows. In order to obtain more reliable source localisation during the process of astrometric correction, the effect of broad-band energy on the exposure map needs to be avoided. Thus, we first produced the exposure-corrected image, the weighted exposure map, and the weighted PSF map. Finally, we performed wcs_match and wcs_update to match all selected Chandra observations with a reference observation ObsID 1808 separately. We defined the source region of each knot based on

1 http://chandra.si.edu/about/science_instruments.html

2 http://cxc.harvard.edu/sherpa/threads/index.html the X-ray positions in Table 1 of Perlman \& Wilson (2005). The source and background regions are shown in Fig. 1.

\subsection{Time variability}

To study the time variabilities, we extracted the flux for each observation in the $0.3-7 \mathrm{keV}$ energy band and built the light curves for each region.

\subsubsection{Nucleus}

The nucleus reveals significant variabilities as shown in the top panel of Fig. 2. We defined five flaring periods, labelled 1-5, and present zoomed-in light curves in Fig. 2. To investigate the characteristic timescales of these flares in more detail, we fitted the light curves with an exponential function of the form

$\Phi=\Phi_{0} \times \mathrm{e}^{-\left|t-t_{0}\right| / \Delta \tau}$.

Here $\Delta \tau=\tau_{\mathrm{d}} / \ln (2), \tau_{\mathrm{d}}$ is the characteristic timescales of each flare. We froze $t_{0}$ to the time of the highest data point for each flare, and $\Phi_{0}$ and $\tau_{\mathrm{d}}$ are free parameters. In principle, the rising and decay timescale can be different. The current data set, however, prevents us from deriving the differences of the two timescales due to the limited time coverage, especially in the rising stage. Thus, for flares 1, 2, and 4 we performed fits assuming that both the rising and decaying stage have the same $\tau_{\mathrm{d}}$. For flares 3 and 5 we only fit the decaying stage. The corresponding values of $\Phi_{0}, \tau_{\mathrm{d}}$, and $t_{0}$ are listed in Table 1 . The characteristic timescale for flare 5 is shortest with $\tau_{\mathrm{d}}=3.9 \pm 1.7$ days. This flare is contemporaneous to the rapid $\mathrm{TeV} \gamma$-ray flare seen on April 910, 2010 (MJD 55296; see e.g. Abramowski et al. 2012; Harris et al. 2011). The day-scale activity seen at X-ray and TeV energies in this context favours a common physical origin of this emission (Abramowski et al. 2012; Aliu et al. 2012).

\subsubsection{HST-1}

HST-1 shows significant variability over time, revealing flux variations much larger than those of the nucleus. During the period from 2002 to 2010 the total X-ray energy flux varied by one order of magnitude, reaching an extreme high state in 2005 (see Fig. 3).

The 2005 X-ray high state of HTS-1 seemingly correlates with a high state of M87 at TeV energies exhibiting rapid day-scale activity (Aharonian et al. 2006). Early models thus assumed a common physical origin, while the apparent absence of such a correlation for the subsequent $\mathrm{TeV}$ high state in 2010 has been taken as disfavouring it. We note that there are several reasons why day-scale TeV activity related to HST-1 now appears disfavoured (see Rieger \& Aharonian 2012, for review and discussion). The presence or absence of a possible X-ray $\mathrm{TeV}$ correlation, however, seems rather less conclusive in this regard as changes in the magnetic field and radiating particle number in a synchrotron (X-ray) and inverse Compton (IC; TeV) approach could fully accommodate both of them.

The X-ray flux of HST-1 seems to be continuously decreasing since 2007 with a characteristic decay timescale of $\sim(0.5-$ 1) yr. This supports previous indications (Harris et al. 2009), and while dominant IC cooling is not excluded (e.g. Perlman et al. 2011), seems compatible with the synchrotron cooling timescale of electrons producing $\sim 1 \mathrm{keV}$ photons in a milligauss magnetic field, $t_{\text {syn }} \lesssim 1.5\left(2 \times 10^{7} / \gamma^{\prime}\right)\left(1 \mathrm{mG} / \mathrm{B}^{\prime}\right)^{2} \delta^{-1} \mathrm{yr}$. This could be taken as providing some additional evidence for a synchrotron origin of the X-ray emission. 

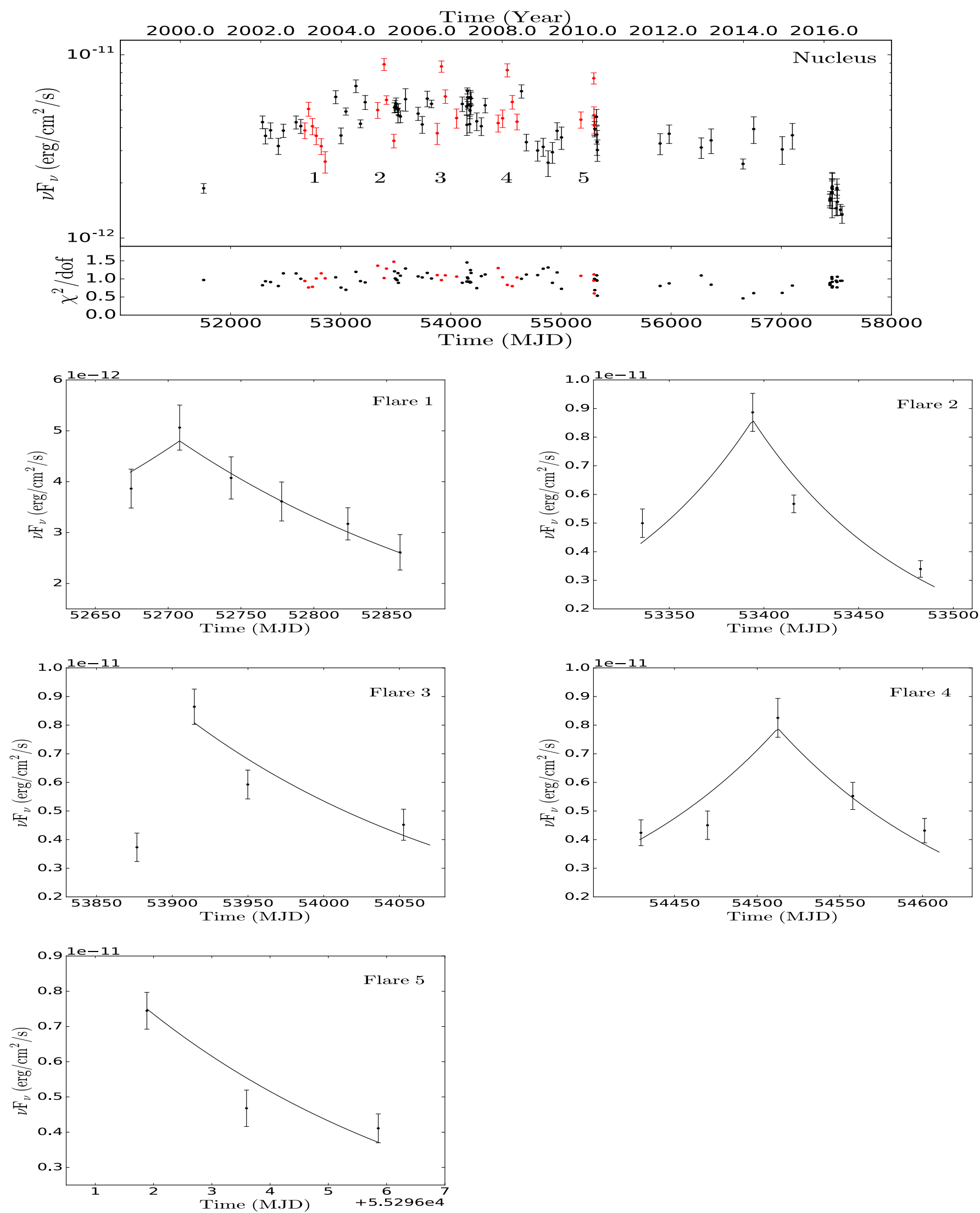

Fig. 2. Top: Chandra 0.3-7 keV light curve for the nucleus from July 29, 2000 (MJD 51754), to March 17, 2016 (MJD 57464). Five flares (red data points, labelled 1-5) are selected for further analysis in the following. Bottom: zoomed-in light curves of the five flares. The solid lines show the fitting results by using Eq. (1). 


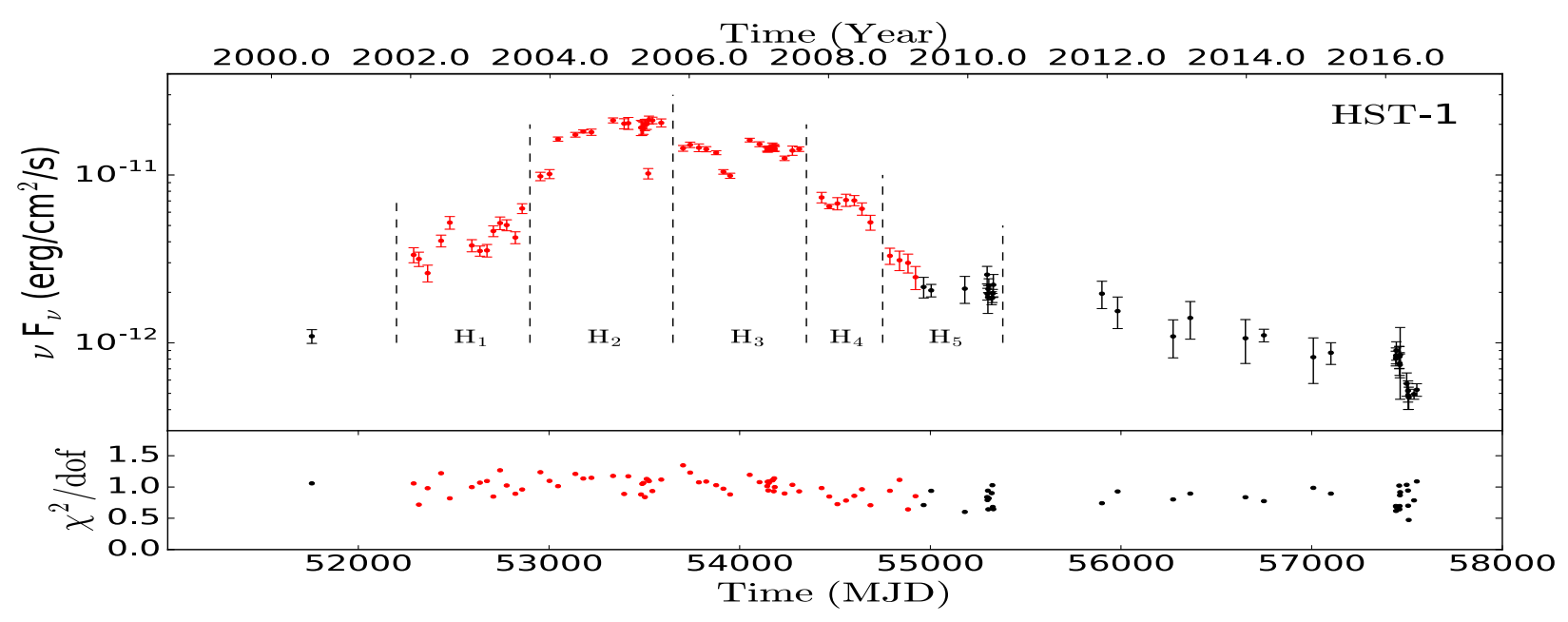

Fig. 3. Chandra 0.3-7 keV light curve for HST-1 during the same time interval as in Fig. 2. The black points are considered to belong to the low state (L). The dashed lines, on the other hand, separate the high state from January 16, 2002 (MJD 52290), to May 14, 2010 (MJD 55330), into five subsections, that are labelled $\mathrm{H}_{1}, \mathrm{H}_{2}, \mathrm{H}_{3}, \mathrm{H}_{4}$, and $\mathrm{H}_{5}$. The data from April 01, 2009 to May 14, 2010, belong to a transition period between high and low state, and have thus been included in both $\mathrm{L}$ and $\mathrm{H}_{5}$ calculations.

Table 1. Fitting parameters for the selected flares of the nucleus using a symmetric exponential function.

\begin{tabular}{lcccc}
\hline \hline Flare & $\begin{array}{c}\text { Time range } \\
(\mathrm{MJD})\end{array}$ & $\begin{array}{c}\tau_{\mathrm{d}} \\
(\text { days })\end{array}$ & $\begin{array}{c}t_{0} \text { (frozen) } \\
(\mathrm{MJD})\end{array}$ & $\begin{array}{c}\Phi_{0} \\
\left(\times 10^{-12} \mathrm{erg} \mathrm{cm}^{-2} \mathrm{~s}^{-1}\right)\end{array}$ \\
\hline Flare 1 & $52675-52859$ & $171.27 \pm 23.07$ & 52708 & $4.80 \pm 0.17$ \\
Flare 2 & $53336-53483$ & $66.36 \pm 16.32$ & 53394 & $8.35 \pm 0.75$ \\
Flare 3 & $53877-54053$ & $143.38 \pm 64.76$ & 53915 & $8.08 \pm 0.97$ \\
Flare 4 & $54430-54601$ & $85.11 \pm 18.95$ & 54512 & $7.89 \pm 0.68$ \\
Flare 5 & $55181-55302$ & $3.91 \pm 1.70$ & 55298 & $7.23 \pm 0.96$ \\
\hline
\end{tabular}

Notes. All errors are at a $1 \sigma$ confidence level.

For the analysis of the spectral variations of HST-1 presented in Sect. 2.3, we divide the high-activity period from January 16, 2002 (MJD 52290), until May 14, 2010 (MJD 55330), into five sections denoted by $\mathrm{H}_{1}, \mathrm{H}_{2}, \mathrm{H}_{3}, \mathrm{H}_{4}$, and $\mathrm{H}_{5}$. The observation on July 30, 2000 (ObsID 1808), and the observations after May 13, 2009 (ObsID 10286), are treated as low state (L). The observations from April 01, 2009 to May 14, 2010, on the other hand, belong to a transition period between the high and the low state, and have thus been included in the following in both, $\mathrm{L}$ and $\mathrm{H}_{5}$ spectral calculations. For a bright source, pile-up could lead to distortions in the energy spectrum (e.g. Harris et al. 2006). Using the pileup_map tool, we estimated the pile-up fraction and found that for $\mathrm{H}_{2}, \mathrm{H} 3$, and $\mathrm{H} 4$ the fraction is larger than $10 \%$, even though we have only selected observations with a frame time of $0.4 \mathrm{~s}$ to avoid pile-up. Thus, for the following spectral analysis we only include H1, H5, and L for HST-1.

\subsubsection{Other knots}

Knot D and knot A show no significant variability. The flux variations lie within the statistical errors of the flux determination, which is $15 \%$ for knot $\mathrm{D}$ and $5 \%$ for knot $\mathrm{A}$. The fluxes derived for knots $\mathrm{E}, \mathrm{F}, \mathrm{I}, \mathrm{B}$, and $\mathrm{C}$ have significant uncertainties due to the limited statistics of the observations. We do not find any evidence of variability even after re-binning the observations. With the exception of HST-1, we thus combined all observations for the following spectral analysis of the knots.

\subsection{Spectral analysis}

For the spectral analysis we performed an aperture photometry using specextract on the nucleus and each knot. The source and background regions are as defined in Fig. 1.

\subsubsection{Nucleus and HST-1 during low states and in early 2010}

The nucleus is highly variable and suffers from pile-up even for observations with frame times of $0.4 \mathrm{~s}$ during the flare peak period $\mathrm{H} 2, \mathrm{H} 3$, and $\mathrm{H} 4$, which could affect the inherent spectral energy distribution (e.g. lead to spectral hardening). Thus, we only use the observations during the period of $\mathrm{H} 1, \mathrm{H} 5$, and L for temporal spectral analyses of nucleus and knot HST-1. To improve the statistics, we combine the observations on time ranges of a few months up to one and a half years. We simultaneously fit the spectra in each time range using a single power law plus Galactic absorption model. To suitably deal with the pile-up we add an additional pile-up model jdpileup to the spectral fit. The time bins and the fitted indices are listed in Table 2. No obvious spectral variability seems apparent. In addition, we also provide a detailed analysis of those observations contemporaneous to the $2010 \mathrm{TeV}$ flare. In $2010 \mathrm{HST}-1$ was back to a state comparable with the pre-2004 time, during which pile-up was found to be fairly mild (Harris et al. 2006; Russell et al. 2015). The fitted indices for the nucleus in April/May 2010 are shown in Table 3 along with those for HST-1. The photon indices for the 

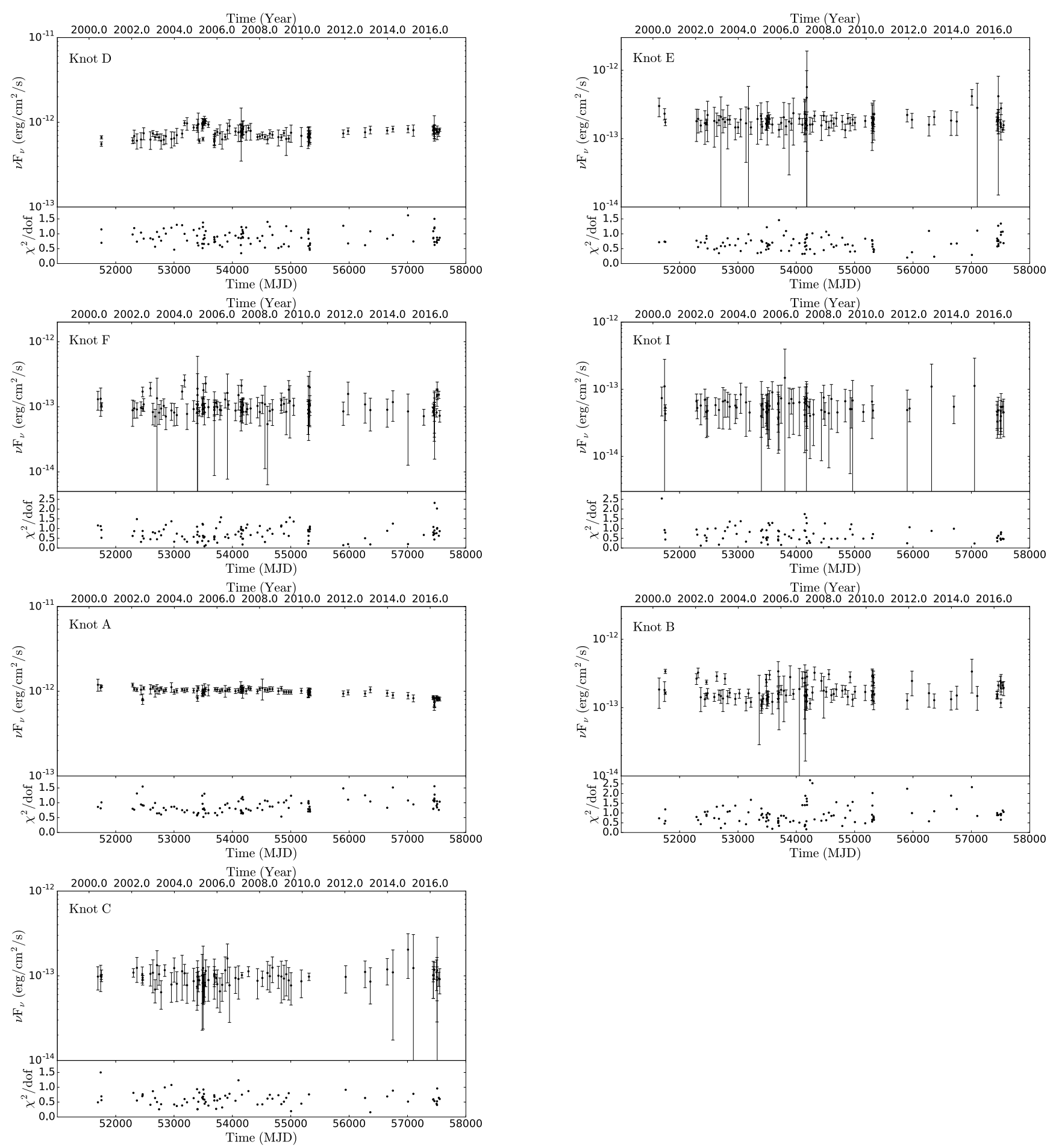

Fig. 4. Chandra 0.3-7 keV light curves for knots D, E, F, I, A, B, and C during the same time interval as in Fig. 2.

nucleus are on average close to $\Gamma \sim 2.1-2.2$, which seems compatible with earlier (non-flaring) results reported in Wilson \& Yang (2002) and Perlman \& Wilson (2005), see also the results in Table 2. A hint for spectral variations during 2010 might be seen in the nucleus, while the HST-1 spectrum appears stable, though the short exposures limit inferences. Combing observations for HST-1 (in a set of three) to improve statistics, however, yields results that are compatible with no spectral change. Our values for the nucleus are similar to those in Harris et al. (2011). Our results for HST-1, however, are harder than those reported in Harris et al. (2011), yet still compatible within the $1 \sigma$ error bars. We note that in our analysis the error bars are much larger due to the additional free parameters in the pile-up model that has been included in our analysis. For sources closer than one arcsec, such as the nucleus and HST-1 (which are separated by $0.86^{\prime \prime}$ ), an "eat-thy-neighbour" effect may in principle occur (Harris et al. 2006, 2009), where photons arriving within the same frame time and $3 \times 3$ pixel grid are registered as a single event at the location of the pixel with the larger energy. As suggested by Harris et al. (2009) this effect is not expected to cause serious problems below a detector-based intensity limit of $4 \mathrm{keV} \mathrm{s}^{-1}$ (e.g. for the pre-2004 and post-2006 time for HST-1). 
Table 2. X-ray photon indices for the nucleus and HST-1 during the period of $\mathrm{H} 1, \mathrm{H} 5$, and $\mathrm{L}$.

\begin{tabular}{ccc}
\hline \hline Time range (YYY-MM-DD) & $\Gamma_{\text {Nucleus }}$ & $\Gamma_{\text {HST-1 }}$ \\
\hline 2002-01-16-2003-08-08 & $2.12 \pm 0.05$ & $2.25 \pm 0.05$ \\
2008-11-17-2010-05-14 & $2.25 \pm 0.05$ & $2.38 \pm 0.07$ \\
2011-12-04-2013-03-12 & $2.27 \pm 0.12$ & $2.52_{-0.28}^{+0.48}$ \\
2013-12-26-2015-03-19 & $2.05 \pm 0.13$ & $2.43_{-0.15}^{+0.30}$ \\
2016-02-23-2016-06-12 & $2.25 \pm 0.10$ & $2.29_{-0.10}^{+0.17}$ \\
\hline
\end{tabular}

Notes. All errors are at a 90\% confidence level.

Table 3. X-ray photon indices for the nucleus and HST-1 during the $2010 \mathrm{TeV}$ flare.

\begin{tabular}{ccc}
\hline \hline ObsID & $\Gamma_{\text {Nucleus }}$ & $\Gamma_{\text {HST }-1}$ \\
\hline 11512 & $1.97 \pm 0.1$ & $2.17_{-0.21}^{+0.24}$ \\
11513 & $2.14_{-0.14}^{+0.15}$ & $2.20_{-0.12}^{+0.27}$ \\
11514 & $1.98_{-0.15}^{+0.16}$ & $2.33_{-0.16}^{+0.31}$ \\
11515 & $2.10_{-0.16}^{+0.17}$ & $2.23_{-0.11}^{+0.18}$ \\
11516 & $1.84_{-0.13}^{+0.16}$ & $2.24_{-0.13}^{+0.26}$ \\
11517 & $2.26_{-0.15}^{+0.16}$ & $2.23_{-0.12}^{+0.28}$ \\
11518 & $2.16_{-0.18}^{+0.20}$ & $2.28_{-0.14}^{+0.18}$ \\
11519 & $2.19_{-0.14}^{+0.19}$ & $2.22_{-0.11}^{+0.21}$ \\
11520 & $2.24_{-0.18}^{+0.21}$ & $2.33_{-0.11}^{+0.29}$ \\
\hline
\end{tabular}

Notes. All errors are at a 90\% confidence level.

The X-ray photon indices for the nucleus are in principle in the range of those achievable by Comptonisation in a hot accretion flow. Early models in fact assumed that the quiescent nuclear $\mathrm{X}$-ray emission in M 87 is produced by an ADAF (e.g. Reynolds et al. 1996; Di Matteo et al. 2003). The similarities of the nuclear spectrum to that of the jet and its knots, and the strong brightness increase towards the nucleus (see Fig. 5), however, suggest that this "nuclear" emission is instead dominated by the jet (Wilson $\&$ Yang 2002). This is supported by the fact that the luminosity of a hot accretion flow at low accretion rates $\dot{M}$ roughly scales with $\dot{M}^{2}$, i.e. $L_{\mathrm{ADAF}} \propto \dot{M}^{2}$, while that of the jet with $L_{\mathrm{j}} \propto \dot{M}$, so that for low $\dot{M}$ the jet starts to dominate (Yuan \& Narayan 2014; Feng \& Wu 2017). The evidence for strong nuclear activity over time fits well into this.

\subsubsection{Combined X-ray spectra of the knots}

For all knots, except HST-1, we fit multiple observations simultaneously. For HST-1 we exclude observations during $\mathrm{H}_{2}, \mathrm{H}_{3}$, and $\mathrm{H}_{4}$ in the spectral analysis due to significant pile-up.

In the X-ray band all knots can in principle be well fitted with a single power law plus Galactic absorption model, none seems to require an obvious break or additional component within the X-ray band itself (but see below). Table 4 lists the best-fit parameter values and the reduced $\chi^{2}$ value. The reduced $\chi^{2}$ values reveal that all fits are acceptable at a $90 \%$ confidence level. Leaving the absorption column density $N_{\mathrm{H}}$ free in the fit yields values deviating significantly from the Galactic one (Perlman \& Wilson 2005). Harris et al. (2006) have reported a mutual dependence between $N_{\mathrm{H}}$ and $\alpha$ in the fitting process, leading to some uncertainty. For comparison, we thus also provide results with $N_{\mathrm{H}}$ frozen to the Galactic value. We note that we do not find evidence for a significant deviation (Osone 2017) from a pure power law in the X-rayband for knot $\mathrm{A}$ if $N_{\mathrm{H}}$ is kept frozen.

The derived photon indices for different knots are significantly different, ranging from $\simeq 1.89($ knot $\mathrm{C})$ to $\simeq 2.61($ knot F $)$. The results roughly match those obtained by Wilson \& Yang (2002, 2004) and Perlman \& Wilson (2005). In Fig. 5 we plot the profiles of the flux density and photon index $\alpha_{\mathrm{x}}$ along the jet. For the regions of the nucleus, knots HST-1, D, and A, we define the size of the stripes of $0.45^{\prime \prime}$ and $2.0^{\prime \prime}$ along and perpendicular to the jet. For the other regions, we use $0.45^{\prime \prime} \times 1.5^{\prime \prime}$ rectangular regions correspondingly. If the signals are too weak, we combine several strips together. We again used the single power law plus Galactic absorption model to fit each region. Finally, we used the best-fit model to simulate the energy flux of the deconvolved non-thermal emission. In this process we divided the $\mathrm{X}$-ray energy interval between $0.3 \mathrm{keV}$ and $7 \mathrm{keV}$ into four bins in logarithmic space. The derived X-ray data points are listed in Table 5. With the exemption of the outermost knot C, the Xray photon index along the jet exhibits a trend similar to that reported in Perlman \& Wilson (2005), with slight but significant index variations ranging from $\simeq 2.2$ (e.g. in knot $\mathrm{D}$ ) to $\simeq 2.4-2.6$ (in knots $\mathrm{F}, \mathrm{A}$, and $\mathrm{B}$ ). There is little evidence that the inter-knot regions have significantly steeper spectra than the adjacent knots, reinforcing the need for a distributed acceleration mechanism.

According to Fig. 5 the X-ray flux density along the jet exhibits significant changes on comparably small scales around the knot D and knot A regions. Radio and optical observations indicate that the knot $\mathrm{D}$ region is in fact composed of several subregions (e.g. Perlman \& Wilson 2005; Avachat et al. 2016, see also below). Our modelling of the broad-band SED of the knots in Sect. 3, on the other hand, suggests that for knot regions B and $\mathrm{C}$, and to some (weaker) extent also for knot region $\mathrm{D}$, an additional X-ray emitting component is needed to achieve a satisfactory SED fit. It seems conceivable that these X-ray features are related to a blending of unresolved longitudinal or radial substructures (i.e. related to lateral jet stratification).

\section{SED modelling}

In order to gain further insights, we constructed the radio to $\mathrm{X}$-ray SEDs of the knots and provided model fits to infer the characteristics of the parent particle distribution. We selected contemporaneous multiwavelength data to ensure an adequate reconstruction. The optical to X-ray spectra of the $\mathrm{H}_{1}, \mathrm{H}_{5}$, and L state of knot HST-1 are taken from the 2002 December, 2003 February, and 2003 April HST observations in Perlman et al. (2003), respectively. The radio data are from Giroletti et al. (2012) based on observations between 2006 and 2010 with the Very Long Baseline Array (VLBA) at $1.7 \mathrm{GHz}$ for $\mathrm{H}_{1}$, and between 2009 and 2011 with the European VLBI Network $(\mathrm{EVN})$ at $5 \mathrm{GHz}$ for the statuses $\mathrm{H}_{5}$ and $\mathrm{L}$. We also used the published optical-near-infrared data from Perlman et al. (2001), and the ultraviolet data from Waters \& Zepf (2005) for knots D, $\mathrm{E}, \mathrm{F}, \mathrm{I}, \mathrm{A}, \mathrm{B}$, and $\mathrm{C}$. The black points in the radio energy band are from Perlman et al. (2001). We did not include the radio data points of Biretta et al. (1991) in the spectral fitting, yet regarded them as a reference for the radio spectral indices because of the uncertainties with regard to these measurements 


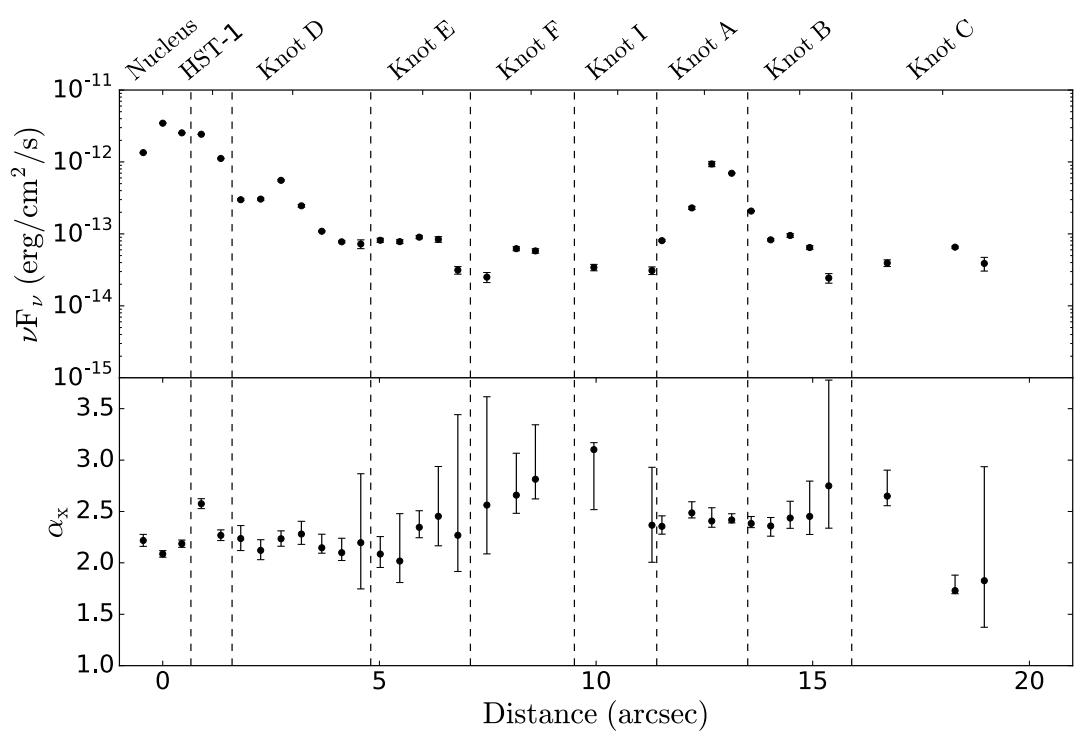

Fig. 5. Flux density (top panel) and power-law photon index (bottom panel) in the X-ray $0.3-7 \mathrm{keV}$ band along the jet. The source regions are separated by the dashed lines. For this representation all observations with frame time $0.4 \mathrm{~s}$ are used for the nucleus, while for HST- 1 only "HST- 1 L" data are employed.

Table 4. Results for the X-ray spectral fitting of the knots.

\begin{tabular}{ccccccc}
\hline \hline Component & $\Gamma^{a}$ & $\begin{array}{c}N_{\mathrm{H}}^{b} \\
\left(\times 10^{20} \mathrm{~cm}^{-2}\right)\end{array}$ & $\begin{array}{c}K^{c} \\
\left(\times 10^{-5} \text { photons } \mathrm{kV}^{-1} \mathrm{~s}^{-1}\right)\end{array}$ & $\begin{array}{c}v \mathrm{~F}_{v}^{\mathrm{d}} \\
\left(\times 10^{-13} \mathrm{erg} \mathrm{cm}^{-2} \mathrm{~s}^{-1}\right)\end{array}$ & reduced $\chi^{2}$ & d.o.f. \\
\hline HST-1 H & $2.25 \pm 0.05$ & $4.74_{-0.75}^{+0.78}$ & $94.88_{-3.10}^{+3.20}$ & $44.73 \pm 0.62$ & 0.98 & 2174 \\
HST-1 H & $2.31 \pm 0.06$ & $1.21_{-1.10}^{+1.15}$ & $50.59_{-2.16}^{+2.45}$ & $23.62 \pm 0.59$ & 0.93 & 1293 \\
HST-1 L & $2.27_{-0.04}^{+0.06}$ & $<1.21$ & $14.53_{-0.35}^{+0.69}$ & $6.86 \pm 0.15$ & 1.08 & 1801 \\
D & $2.22 \pm 0.03$ & $2.50 \pm 0.5$ & $17.11 \pm 0.4$ & $8.13 \pm 0.10$ & 0.72 & 5526 \\
E & $2.35 \pm 0.05$ & $2.34_{-0.83}^{+0.86}$ & $4.10_{-0.15}^{+0.16}$ & $1.91 \pm 0.04$ & 0.70 & 2726 \\
F & $2.61_{-0.02}^{+0.07}$ & $<0.46$ & $2.15_{-0.05}^{+0.06}$ & $1.00 \pm 0.01$ & 0.74 & 1720 \\
I & $2.50_{-0.11}^{+0.04}$ & $<0.57$ & $1.20_{-0.08}^{+0.02}$ & $0.56 \pm 0.04$ & 1.02 & 576 \\
A & $2.41 \pm 0.02$ & $<0.10$ & $21.22_{-0.36}^{+0.13}$ & $9.85 \pm 0.02$ & 0.90 & 4811 \\
B & $2.35_{-0.02}^{+0.06}$ & $<0.8$ & $3.49_{-0.06}^{+0.13}$ & $1.63 \pm 0.02$ & 0.89 & 2480 \\
C & $1.89_{-0.03}^{+0.09}$ & $<0.85$ & $1.78_{-0.04}^{+0.12}$ & $0.94 \pm 0.02$ & 0.64 & 2145 \\
\hline F & $2.79 \pm 0.04$ & 2.4 (frozen) & $2.36 \pm 0.05$ & $1.13 \pm 0.02$ & 0.75 & 1721 \\
I & $2.60 \pm 0.04$ & 2.4 (frozen) & $1.28 \pm 0.03$ & $0.60 \pm 0.01$ & 1.04 & 577 \\
A & $2.55 \pm 0.01$ & 2.4 (frozen) & $23.10 \pm 0.2$ & $10.74 \pm 0.05$ & 0.96 & 4812 \\
B & $2.49 \pm 0.03$ & 2.4 (frozen) & $3.85 \pm 0.06$ & $1.79 \pm 0.02$ & 0.90 & 2481 \\
C & $2.01 \pm 0.04$ & 2.4 (frozen) & $1.99 \pm 0.05$ & $1.0 \pm 0.02$ & 0.65 & 2146 \\
\hline
\end{tabular}

Notes. All errors are at a $90 \%$ confidence level. $\mathrm{H}_{1}, \mathrm{H}_{5}$, and L are defined as shown in Fig. 3. ${ }^{(a)}$ Photon index. ${ }^{(b)}$ Equivalent hydrogen-absorbing column density. ${ }^{(c)}$ Amplitude of power-law model. ${ }^{(d)}$ Total integrated energy flux over $0.3-7 \mathrm{keV}$. The bottom part provides the results if $N_{\mathrm{H}}$ is frozen to the Galactic value.

(see e.g. Perlman \& Wilson 2005). It should also be noted that although our region $\mathrm{C}$ could be further divided into the subregions $\mathrm{C} 1$ and $\mathrm{C} 2$, we only include the ultraviolet fluxes for $\mathrm{C} 1$. This simplification appears justified considering the much lower ultraviolet flux from $\mathrm{C} 2$. For comparison, we also plot the Xray flux density from Marshall et al. (2002) with grey symbols in Fig. 6.

There are in principle several possible non-thermal scenarios for the origin of large-scale X-ray emission, such as inverse Compton (IC) upscattering of either synchrotron (synchrotron self-Compton; IC-SSC) or cosmic microwave background (IC-CMB) photons, or direct synchrotron emission of relativistic electrons or protons (e.g. Aharonian 2002; Harris \& Krawczynski 2002; Zhang et al. 2009, 2010; Georganopoulos et al. 2016). As discussed in Wilson \& Yang (2002), to account for the keV X-ray flux by means of leptonic IC-SSC processes, the magnetic field has to be substantially lower than equipartition. In the case of IC-CMB it is possible to boost the X-ray flux 
Table 5. Deconvolved X-ray flux densities of the knots for different energy bands.

\begin{tabular}{ccccc}
\hline \hline Energy [keV] & $0.3-0.7$ & $0.7-1.4$ & $1.4-3.2$ & $3.2-7.0$ \\
\hline Component & \multicolumn{3}{c}{ Flux density $\left[\times 10^{-14} \mathrm{erg} \mathrm{cm}^{-2} \mathrm{~s}^{-1}\right]$} \\
\hline HST-1 H & $159.06 \pm 3.23$ & $106.78 \pm 1.47$ & $104.89 \pm 2.10$ & $81.78 \pm 2.64$ \\
HST-1 H & $89.26 \pm 3.11$ & $56.74 \pm 1.29$ & $53.78 \pm 1.68$ & $39.83 \pm 2.33$ \\
HST-1 L & $24.58 \pm 0.97$ & $16.40 \pm 0.40$ & $15.92 \pm 0.66$ & $12.14 \pm 0.97$ \\
D & $23.03 \pm 0.33$ & $17.22 \pm 0.17$ & $18.71 \pm 0.24$ & $16.10 \pm 0.33$ \\
E & $7.47 \pm 0.20$ & $4.61 \pm 0.10$ & $4.21 \pm 0.12$ & $3.02 \pm 0.14$ \\
F & $4.82 \pm 0.11$ & $2.44 \pm 0.03$ & $1.83 \pm 0.04$ & $1.05 \pm 0.04$ \\
I & $2.48 \pm 0.26$ & $1.36 \pm 0.09$ & $1.12 \pm 0.13$ & $0.69 \pm 0.12$ \\
A & $40.38 \pm 0.19$ & $23.94 \pm 0.06$ & $20.86 \pm 0.09$ & $14.19 \pm 0.12$ \\
B & $6.33 \pm 0.10$ & $3.93 \pm 0.05$ & $3.58 \pm 0.10$ & $2.56 \pm 0.05$ \\
C & $2.25 \pm 0.04$ & $2.00 \pm 0.03$ & $2.59 \pm 0.06$ & $2.67 \pm 0.10$ \\
\hline
\end{tabular}

by relativistic bulk motion, but this requires high Doppler factors $\delta \simeq(10-40)$ and very small angles $\theta \simeq\left(1.2^{\circ}-4.7^{\circ}\right)$ between the jet and the line of sight (Harris \& Krawczynski 2002). Though HST observations indicate that moderate superluminal motion in M 87 could persist out to knot C (Meyer et al. 2013), such values remain highly unlikely. Moreover, IC radiation by a power-law electron distribution in the Thomson regime is expected to exhibit a spectral index similar to that for synchrotron radiation, yet the observed X-ray spectra of the knots are all significantly steeper than the radio spectra (exhibiting spectral indices $\alpha_{\mathrm{rr}} \sim 0.5$, e.g. Biretta et al. 1991). An IC origin of the Xray emission in M 87 thus appears disfavoured (see also Biretta et al. 1991; Meisenheimer et al. 1996; Wilson \& Yang 2002; Perlman \& Wilson 2005; Zhang et al. 2010), and we therefore only consider an electron synchrotron origin in the following.

We use Naima (Zabalza 2015) to explore the characteristics of the radiating particle distribution. Naima is a numerical package that includes a set of non-thermal radiative models and a spectral fitting procedure. The best-fit and uncertainty distributions of spectral model parameters are derived through Markov chain Monte Carlo (MCMC; Foreman-Mackey et al. 2013) sampling of their likelihood distributions. The code allows us to implement different functions and includes tools to perform MCMC fitting of non-thermal radiative processes to the data. Given the radio to X-ray data of the knots in M 87, the simplest functional form to take into account is a broken power law,

$$
N(E)= \begin{cases}A E^{-\alpha_{1}} & E \leq E_{\text {break }} \\ A E_{\text {break }}^{\left(\alpha_{2}-\alpha_{1}\right)} E^{-\alpha_{2}} & E>E_{\text {break }} .\end{cases}
$$

For the fitting process, the parameters $A, \alpha_{1}, E_{\text {break }}$, and $\alpha_{2}$ are left as free parameters and we assume non-relativistic motion for the entire jet. Our results (Table 6) show that the SEDs of knots E, F, I, and A could in principle be satisfactorily described by synchrotron emission of a broken power-law electron distribution with an index $\alpha_{1} \simeq 2.3$ resembling that of particle acceleration at highly relativistic shocks (e.g. Achterberg et al. 2001). The second index $\alpha_{2}$ for these knots deviates from that of a classical cooling break. This is interesting, but could possibly be accounted for by deviations from a homogeneous one-zone approach and/or some additional re-acceleration (e.g. Liu \& Shen 2007; Sahayanathan 2008; Liu et al. 2017). Diffusive synchrotron radiation from random small-scale magnetic fields could offer an alternative explanation (Fleishman 2006), although the observed high radio and optical polarisation (e.g. Avachat et al. 2016) may complicate this interpretation. As an alternative a log-parabola model (cf. Massaro et al. 2004; Tramacere et al. 2007),

$N(E)=A\left(\frac{E}{100 \mathrm{GeV}}\right)^{-\alpha-\beta \log \left(\frac{E}{100 \mathrm{GeV}}\right)}$,

has also been employed to fit the SEDs, and the corresponding results are presented in Table 6. A comparison of the MLL values for a broken power-law and a log-parabola model, using the Bayesian information criterion (BIC), shows that the first is preferred over the second.

The SEDs of knots D, B, and C, on the other hand, cannot be well-fitted assuming a homogeneous source region and the broken power-law from Eq. (2). In principle, this should not come as a surprise given the morphological complexity of the knots and the evidence of internal jet stratification (e.g. Perlman \& Wilson 2005; Avachat et al. 2016). The findings are nevertheless quite interesting and provide a first indication that the X-ray emission of large-scale AGN jets might reveal some excess above a simple power-law extension from the optical fluxes, and in fact consist of multiple contributions. To explore this in more detail, we added an additional electron component to fit the X-ray data. For simplicity we chose a two exponential cut-off power-laws model,

$$
\begin{aligned}
N(E) & =A_{1} E^{-\alpha_{1}} \exp \left[-\left(\frac{E}{E_{\text {cutoff } 1}}\right)^{\beta}\right] \\
& +A_{2} E^{-\alpha_{2}} \exp \left[-\left(\frac{E}{E_{\text {cutoff } 2}}\right)^{\beta}\right],
\end{aligned}
$$

treating again $A_{1}, \alpha_{1}, E_{\text {cutoff1 }}, A_{2}, \alpha_{2}$, and $E_{\text {cutoff2 }}$ as free parameters for the fit. We note that alternative descriptions (e.g. two broken power laws with exponential cut-off) are in principle possible, yet require that some additional parameters be fixed. In our phenomenological fit the two components are treated as independent. We note, though, that the correlation between the X-ray flux maxima and the synchrotron break frequencies (e.g. Perlman \& Wilson 2005) suggest that the X-ray emitting particles are not completely independent from those at lower energies. The results should thus be treated only as a first guide. 

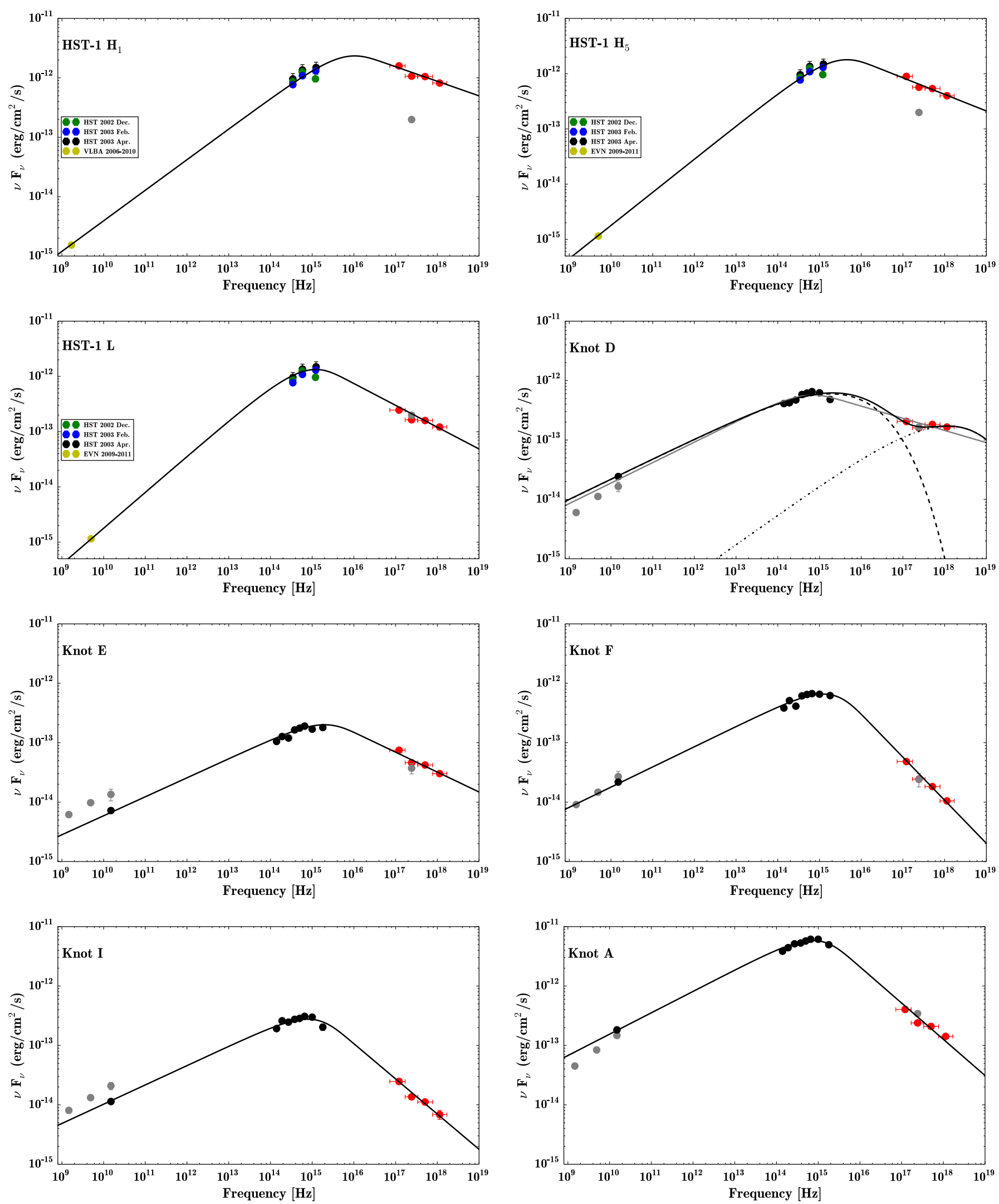

Fig. 6. SEDs fitting results assuming a synchrotron origin of the X-ray emission. The curves represent the best-fit models. For knots D, B, and C both a broken power-law fit and a fit based on two exponential cut-off power-law components (dashed and dot-dashed curves, with superposition corresponding to the solid curve) assuming $\beta=1$ are shown. The X-ray flux densities from Marshall et al. (2002) are also plotted for comparison (grey symbols). 


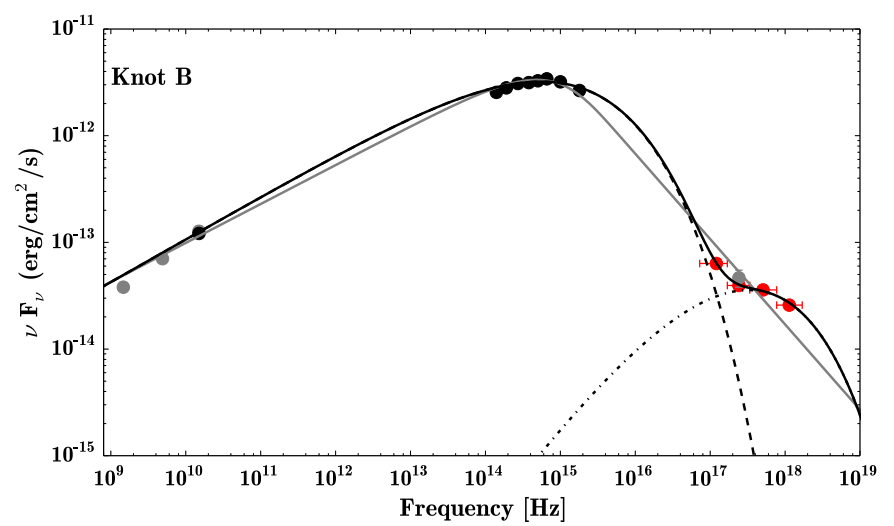

Fig. 6. continued

To reduce the free parameters in our fit (Eq. (4)), we fixed the parameter $\beta$ to 1 and 2, corresponding to the escaping and cooling-dominated regime, respectively. We assume a magnetic field strength of $B=300 \mu \mathrm{G}$ (Heinz \& Begelman 1997; Marshall et al. 2002) as a reference value in all our calculations. This is certainly a simplification as the magnetic field strength is expected to be different for different regions, for example it can vary by a factor of $\sim 2$ when different knots are compared (e.g. Biretta et al. 1991; Meisenheimer et al. 1996). We note however, that in the fitting procedure $B$ scales as $E_{\text {cutoff }}^{-2}$ and $A^{-0.5}$, so that a different $B$ only affects the best-fit value of the break or cut-off energy, and the absolute normalisation.

The derived model parameters as well as the total energy in non-thermal electrons, $W_{\mathrm{e}}$, with $1 \sigma$ errors are listed in Table 6 . Figure 6 shows the corresponding synchrotron model fits for the observed SEDs of the knots. The curves represent the SED models with the maximum likelihood. For knots D, B, and C, the dashed curves and the dot-dashed curves are derived from two exponential cut-off power-law components, respectively, as noted above. The derived values for the required energy in nonthermal electrons range up to $W_{\mathrm{e}} \simeq 10^{53} \mathrm{erg}(\mathrm{knot} \mathrm{A})$ and imply a jet kinetic power $P_{\mathrm{j}} \gtrsim 10^{43} \mathrm{erg} \mathrm{s}^{-1}$, compatible with other estimates for the general jet power (see Introduction). The energy density in electrons, $u_{\mathrm{e}}$, is typically comparable to (or somewhat less than) that in magnetic fields, $u_{B}=B^{2} /(8 \pi)$, ensuring efficient confinement.

Our findings provide indications that for knot regions D, $\mathrm{B}$, and $\mathrm{C}$ an additional $\mathrm{X}$-ray emitting component is needed to achieve a satisfactory SED description. The indications are strongest in the case of knot $\mathrm{C}$ where the $\mathrm{X}$-ray emission seems particularly hard, and less strong in the case of knot D. It is worth noting that radio and optical observations show that the knot $\mathrm{D}$ region, located approximately between 2 and 5 arcsec, reveals extended structures and can be further divided into subregions D-East, D-Middle, and D-West, each with additional subcomponents (e.g. Perlman \& Wilson 2005; Avachat et al. 2016). Similarly, knot B, located approximately between 13 and 16 arcsec, can be divided into the subcomponents B1 (broader and brighter) and $\mathrm{B} 2$, while the knot $\mathrm{C}$ region, located approximately between 17 and 19 arcsec, can be divided into the subregions C1 and C2 (Perlman \& Wilson 2005; Avachat et al. 2016). It seems thus conceivable that the inferred two-component electron distribution can in principle be accounted for by a superposition of electrons from different subregions. Moreover, the features seen in the optical tend to be slightly narrower than those seen in radio, suggesting that the radiating particles, although co-located, may not necessarily have to be truly co-spatial. We

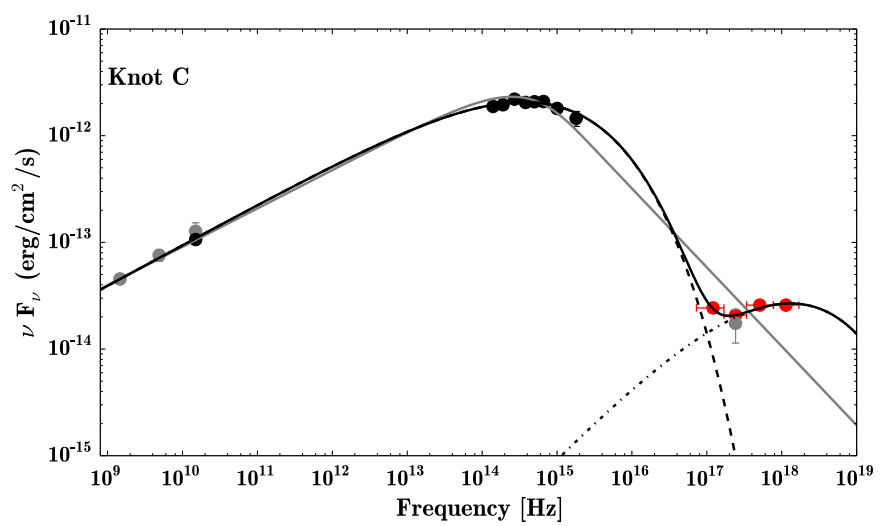

note that we have incorporated only the simplest broken powerlaw function to fit the SED of the knots (except for B, C, and D) and formally cannot rule out the possibility that the SEDs of these knots are produced by more than one electron component as well. Noting these caveats, the respective parent electrons distributions are shown in Fig. 7. As can be seen, for the considered normalisation of the magnetic field strength the electron distribution tends to show a break at energies around one $\mathrm{TeV}$ $\left(\gamma_{b} \sim 2 \times 10^{6}\right)$ and needs to extend up to multi-TeV energies $\left(\gamma \sim 10^{8}\right)$. These high-energy electrons cannot travel more than a few parsecs before exhausting their energies due to synchrotron cooling. A synchrotron origin thus requires an efficient and continuous in situ acceleration of electrons along the jet.

Inverse Compton (IC) up-scattering of cosmic microwave background $(\mathrm{CMB})$ photons $\left(h v_{\mathrm{CMB}}\right)$ by these relativistic electrons will result in $\gamma$-ray emission extending up to $\sim 10\left(\gamma / 10^{8}\right)^{2} \mathrm{TeV}$. The characteristic energy flux levels $v F_{v}$ at $\mathrm{TeV}$ energies are, however, a factor of $\sim u_{C M B} / u_{B} \sim 10^{-3}$ lower than those seen at X-ray energies $\left(\nu F_{v} \lesssim 10^{-12} \operatorname{erg~} \mathrm{cm}^{-1} \mathrm{~s}^{-1}\right)$. This $\mathrm{TeV}$ emission would thus be below the flux sensitivity limit of current $\gamma$-ray instruments.

\section{Conclusions}

We have collected Chandra ACIS data for M 87 between 2000 and 2016 with a total exposure of about $1.5 \mathrm{Ms}$ to perform a temporal and spectral analysis of its nucleus and knots. The extracted X-ray light curves of the nucleus and HST-1 reveal significant variability. We confirm indications for day-scale nuclear Xray variability contemporaneous to the TeV flare in April 2010. HST-1 shows a decline in X-ray flux since 2007 compatible with its synchrotron origin.

The X-ray spectra of the nucleus and jet knots are all formally compatible with a single power law. The resultant X-ray photon index reveals a trend, with index variations ranging from $\simeq 2.2$ (e.g. in knot D) to $\simeq 2.4-2.6$ (in the outer knots $\mathrm{F}, \mathrm{A}$, and $\mathrm{B}$ ). When placed in a multiband context, a more complex situation is seen. Modelling the radio to X-ray SEDs with a synchrotron model, a single broken power-law electron distribution with a break at around $E_{\mathrm{b}} \sim 1 \mathrm{TeV}$ (assuming $B \sim 300 \mu \mathrm{G}$ ) seemingly allows a satisfactorily SED description for most knots. However, for knots B, C, and D an additional high-energy component is needed to account for the broad-band SEDs. This may be partly due to a blending of different (non-resolved) components and/or the occurrence of additional acceleration and emission processes. 
Table 6. SEDs fit results of the knots of M 87.

\begin{tabular}{|c|c|c|c|c|c|c|}
\hline \multicolumn{7}{|c|}{ Broken power-law model } \\
\hline Component & $\begin{array}{c}W_{\mathrm{e}}(>1 \mathrm{GeV}) \\
\left(\times 10^{51} \mathrm{erg}\right)\end{array}$ & $\alpha_{1}$ & $\begin{array}{l}E_{\text {break }} \\
(\mathrm{GeV})\end{array}$ & $\alpha_{2}$ & $M L L^{a}$ & \\
\hline HST-1 $\mathrm{H}_{1}$ & $6.5_{-1.9}^{+1.3}$ & $2.08_{-0.12}^{+0.06}$ & $1300 \pm 60$ & $3.49 \pm 0.07$ & -57 & \\
\hline HST-1 $\mathrm{H}_{5}$ & $3.8 \pm 0.5$ & $1.85_{-0.09}^{+0.06}$ & $900_{-80}^{+60}$ & $3.63 \pm 0.09$ & -28 & \\
\hline HST-1 L & $4.4_{-0.5}^{+0.7}$ & $1.77 \pm 0.07$ & $460_{-30}^{+20}$ & $3.83 \pm 0.08$ & -27 & \\
\hline $\mathrm{E}$ & $3.1_{-0.6}^{+0.4}$ & $2.37_{-0.06}^{+0.03}$ & $680_{-50}^{+40}$ & $3.65 \pm 0.07$ & -42 & \\
\hline $\mathrm{F}$ & $10.1 \pm 1.4$ & $2.33 \pm 0.04$ & $630 \pm 30$ & $4.52 \pm 0.10$ & -79 & \\
\hline I & $5.2 \pm 0.8$ & $2.34_{-0.03}^{+0.04}$ & $470 \pm 20$ & $4.23 \pm 0.08$ & -56 & \\
\hline A & $90_{-8}^{+14}$ & $2.3_{-0.03}^{+0.04}$ & $480 \pm 20$ & $4.25_{-0.08}^{+0.12}$ & -60 & \\
\hline \multicolumn{7}{|c|}{ Two exponential cut-off power-laws model } \\
\hline Component & $\begin{array}{c}W_{\mathrm{e}}(>1 \mathrm{GeV}) \\
\left(\times 10^{51} \mathrm{erg}\right)\end{array}$ & $\alpha_{1}$ & $\begin{array}{l}E_{\text {cutoff1 }} \\
(\mathrm{GeV}) \\
\end{array}$ & $\alpha_{2}$ & $\begin{array}{l}E_{\text {cutoff2 }} \\
(\mathrm{GeV}) \\
\end{array}$ & $M L L^{a}$ \\
\hline $\mathrm{D}(\beta=1)$ & $11.3_{-0.08}^{+0.13}$ & $2.32 \pm 0.01$ & $1130 \pm 40$ & $2.0 \pm 0.10$ & $19400_{-1700}^{2000}$ & -96 \\
\hline $\mathrm{B}(\beta=1)$ & $62.2 \pm 0.6$ & $2.20 \pm 0.01$ & $521 \pm 9$ & $1.05 \pm 0.05$ & $4900 \pm 200$ & -11 \\
\hline $\mathrm{C}(\beta=1)$ & $51.2 \pm 0.4$ & $2.24 \pm 0.01$ & $443 \pm 5$ & $1.51 \pm 0.07$ & $13100 \pm 1100$ & -16 \\
\hline $\mathrm{D}(\beta=2)$ & $11.1 \pm 1.6$ & $2.36 \pm 0.04$ & $1110 \pm 50$ & $2.14 \pm 0.07$ & $16200 \pm 700$ & -202 \\
\hline $\mathrm{B}(\beta=2)$ & $58_{-4}^{+9}$ & $2.33 \pm 0.03$ & $1150_{-60}^{+50}$ & $0.92 \pm 0.04$ & $8000 \pm 400$ & -49 \\
\hline $\mathrm{C}(\beta=2)$ & $49 \pm 5$ & $2.32 \pm 0.03$ & $640 \pm 30$ & $1.75_{-0.05}^{+0.04}$ & $17200 \pm 800$ & -179 \\
\hline \multicolumn{7}{|c|}{ Log-parabola model } \\
\hline Component & $\begin{array}{c}W_{\mathrm{e}}(>1 \mathrm{GeV}) \\
\left(\times 10^{51} \mathrm{erg}\right)\end{array}$ & $\alpha$ & $\beta$ & $M L L^{a}$ & & \\
\hline HST-1 $\mathrm{H}_{1}$ & $11.5 \pm 1.3$ & $2.35 \pm 0.1$ & $0.12 \pm 0.01$ & -82 & & \\
\hline HST-1 $\mathrm{H}_{5}$ & $7.4 \pm 0.7$ & $2.21 \pm 0.1$ & $0.17 \pm 0.01$ & -36 & & \\
\hline HST-1 L & $9.5_{-1.3}^{+0.7}$ & $2.36 \pm 0.06$ & $0.23 \pm 0.01$ & -72 & & \\
\hline $\mathrm{D}$ & $13.5 \pm 1.0$ & $2.67_{-0.03}^{+0.02}$ & $0.12 \pm 0.01$ & -450 & & \\
\hline $\mathrm{E}$ & $4.0_{-0.3}^{+0.4}$ & $2.68_{-0.02}^{+0.03}$ & $0.12 \pm 0.01$ & -133 & & \\
\hline $\mathrm{F}$ & $16.4_{-1.1}^{+2.0}$ & $2.85 \pm 0.03$ & $0.21 \pm 0.01$ & -389 & & \\
\hline $\mathrm{I}$ & $7.8 \pm 0.7$ & $2.85 \pm 0.03$ & $0.21 \pm 0.01$ & -266 & & \\
\hline A & $143 \pm 10$ & $2.82_{-0.02}^{+0.05}$ & $0.21 \pm 0.01$ & -326 & & \\
\hline $\mathrm{B}$ & $106 \pm 9$ & $2.98 \pm 0.04$ & $0.28 \pm 0.01$ & -550 & & \\
\hline $\mathrm{C}$ & $84_{-11}^{+8}$ & $3.07_{-0.06}^{+0.03}$ & $0.28 \pm 0.01$ & -2543 & & \\
\hline
\end{tabular}

Notes. Results of the two-component fit are shown for knots D, B, and C. All errors correspond to a $1 \sigma$ confidence level. ${ }^{(a)}$ Maximum log-likelihood (MML).

The most favourable interpretation for the origin of the $\mathrm{X}$ ray emission is synchrotron radiation of relativistic electrons. This requires that electrons should be able to reach and sustain energies of $\gamma \sim 10^{8}(300 \mu \mathrm{G} / B)^{1 / 2}$ in the presence of losses, i.e. an in situ acceleration of electrons in the jet and its knots. The necessary acceleration efficiency $\eta$, defined by $t_{\mathrm{acc}}=\gamma m_{e} c /(\eta e B)$ (Aharonian et al. 2002), would be of the order of $\eta \gtrsim 3 \times 10^{-4}(B / 300 \mu \mathrm{G})$, and likely to be in the reach of stochastic acceleration scenarios (e.g. Rieger et al. 2007). Localised (first-order Fermi) shock acceleration alone, however, would not be sufficient given that here is little evidence for the inter-knot regions to have significantly steeper spectra than the adjacent knots.
The homogeneous broken power-law model exhibits a change in indices exceeding that induced by simple cooling effects. Assuming a continuous power-law injection, Perlman \& Wilson (2005) have suggested that an energy-dependent filling factor $f_{\text {acc }}(\gamma) \propto \gamma^{-\xi}$ of the acceleration regions (with $\xi \sim 0.3$, i.e. occupying a smaller fraction at higher energies), might account for these breaks. Alternative explanations could be a spatial varied propagation of the relativistic electrons or a different particle injection spectrum in a different energy band. However, to some extent all of these assumptions are ad hoc, and detailed modelling as well as an advanced morphological analysis in different energy bands would be required to further qualify them. 

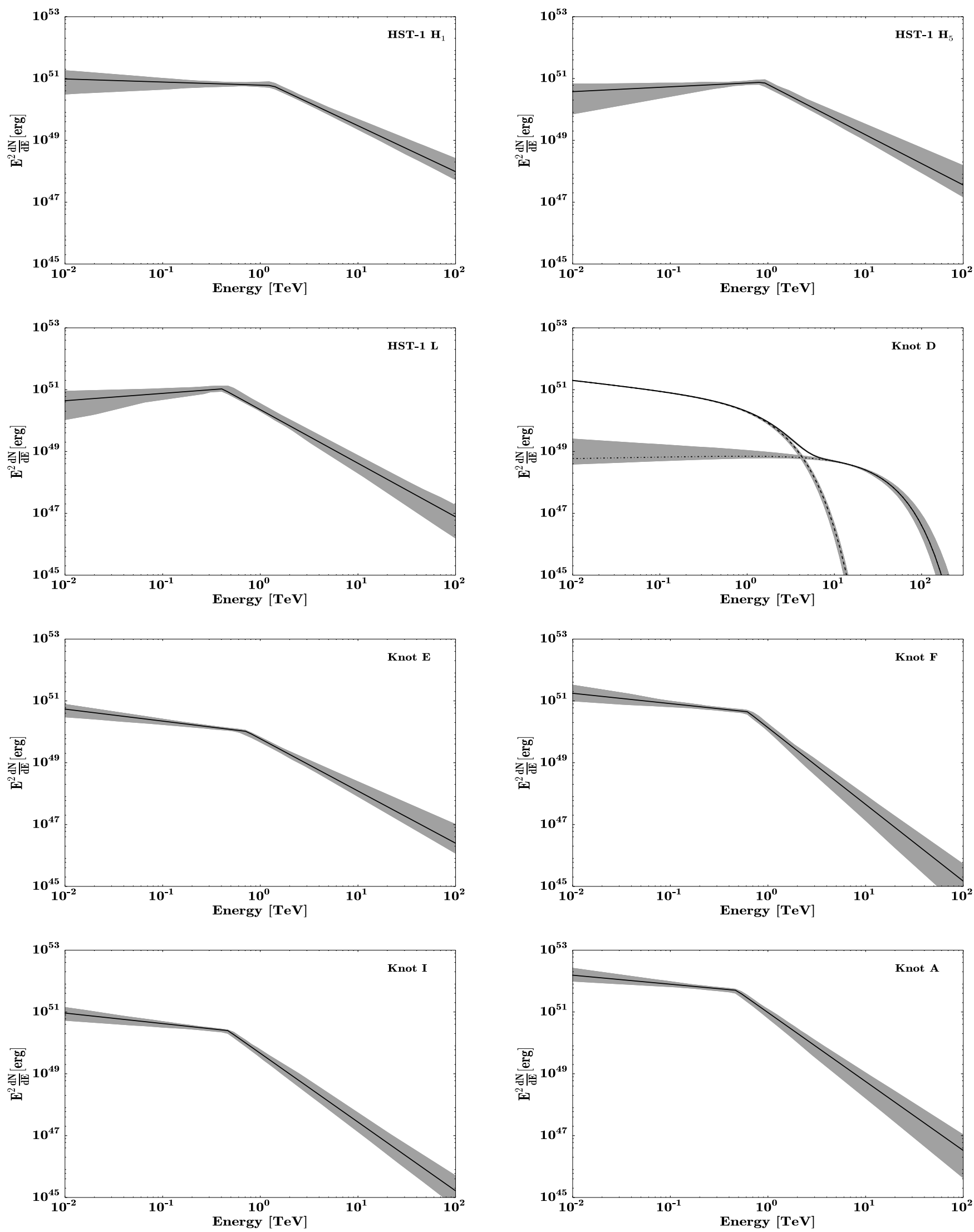

Fig. 7. Parent electron distributions derived from the fitting in Fig. 6. For knots D, B, and C, only the results of the two exponential cut-off power laws are shown. The grey shadow areas correspond to a $2 \sigma$ confidence level. 

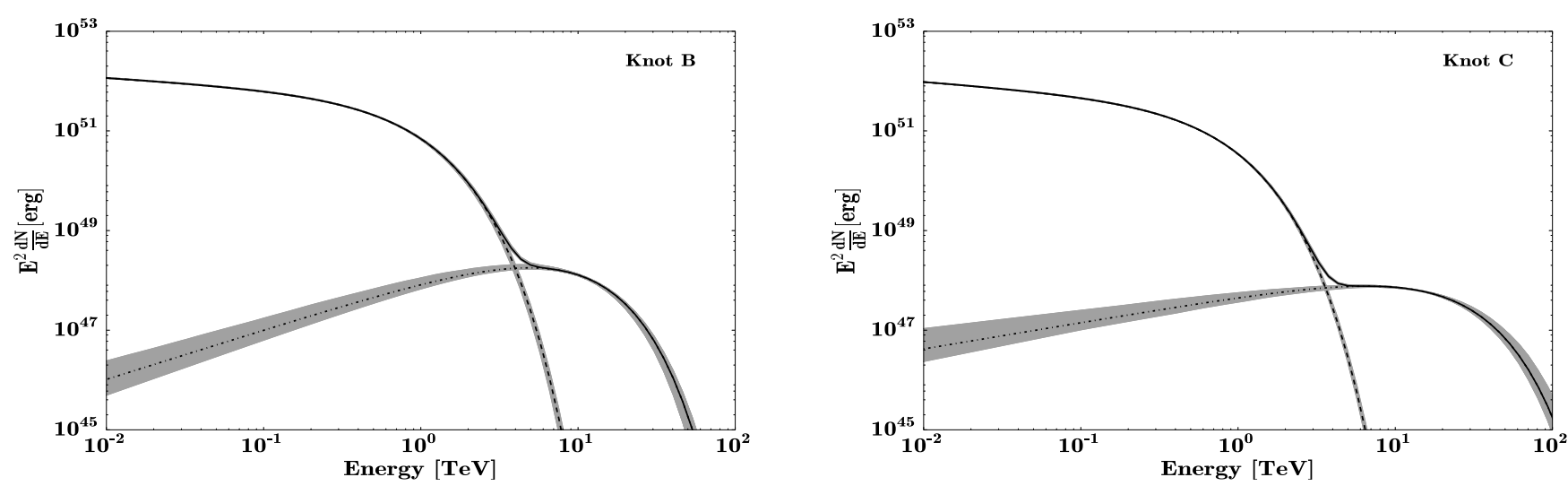

Fig. 7. continued

There are clear indications that in the case of knots B $\mathrm{C}$, and $\mathrm{D}$ an additional electron contribution is needed to account for the X-ray emission in a multiband context. We formally cannot rule out the possibility that the SEDs of other knots are also produced by a two-component (or more) electron distribution, though the smoothness of the fits might seem to argue for the opposite, i.e. there is no evidence that the X-ray emission in these knots consists of separate spectral components.

The additional electron component indicated in the case of knots B, C, and D is consistent with a bump or a spike-like feature at high energy. This appears compatible with a scenario where electron acceleration occurs in a shear layer and radiative loss is effective (Ostrowski 2000). The short cooling time of the relativistic electrons generally requires that in addition to a possible localised shock-type scenario there must be some distributed in situ acceleration occurring along the jet. Stochastic or shear acceleration could present a natural explanation for this (e.g. Liu et al. 2017). There is circumstantial evidence for a decreased thermal gas emissivity along the jet that appears consistent with the dynamical effects of a cocoon dominated by cosmic rays (CR) accelerated at the shearing jet side boundary (Dainotti et al. 2012). The hint of a CR cocoon and the spectral shape of the relativistic electrons in these knots could indicate that efficient shear acceleration indeed is taking place along the jet in M 87, which seems to link well into the broader evidence for a stratified or spine-sheath flow (e.g. Perlman et al. 1999).

Acknowledgements. We thank the referee Eric Perlman for the very useful comments and suggestions that helped to improve the paper. X.N. Sun thanks Jin Zhang for helpful discussions, and acknowledges the financial support of the China Scholar Council. F.M.R. kindly acknowledges support by a DFG Heisenberg Fellowship (RI 1187/4-1). This research has made use of data obtained from the Chandra Data Archive and the Chandra Source Catalog, and software provided by the Chandra X-ray Center (CXC) in the application packages CIAO, ChIPS, and Sherpa.

\section{References}

Abramowski, A., Acero, F., Aharonian, F., et al. 2012, ApJ, 746, 151

Achterberg, A., Gallant, Y. A., Kirk, J. G., \& Guthmann, A. W. 2001, MNRAS, 328,393

Aharonian, F. A. 2002, MNRAS, 332, 215

Aharonian, F. A., Belyanin, A. A., Derishev, E. V., Kocharovsky, V. V., \& Kocharovsky, V. V. 2002, Phys. Rev. D, 66, 023005

Aharonian, F., Akhperjanian, A. G., Bazer-Bachi, A. R., et al. 2006, Science, 314 1424

Aliu, E., Arlen, T., Aune, T., et al. 2012, ApJ, 746, 141
Avachat, S. S., Perlman, E. S., Adams, S. C., et al. 2016, ApJ, 832, 3 Biretta, J. A., Stern, C. P., \& Harris, D. E. 1991, AJ, 101, 1632 Biretta, J. A., Sparks, W. B., \& Macchetto, F. 1999, ApJ, 520, 621 Blakeslee, J. P., Jordán, A., Mei, S., et al. 2009, ApJ, 694, 556 Britzen, S., Fendt, C., Eckart, A., \& Karas, V. 2017, A\&A, 601, A52 Dainotti, M. G., Ostrowski, M., Harris, D., Siemiginowska, A., \& Siejkowski, H. 2012, MNRAS, 426, 218

Davis, J. E. 2001, ApJ, 562, 575

de Gasperin, F., Orrú, E., Murgia, M., et al. 2012, A\&A, 547, A56

Di Matteo, T., Allen, S. W., Fabian, A. C., Wilson, A. S., \& Young, A. J. 2003, ApJ, 582, 133

Doeleman, S. S., Fish, V. L., Schenck, D. E., et al. 2012, Science, 338, 355

Feng, J., \& Wu, Q. 2017, MNRAS, 470, 612

Fleishman, G. D. 2006, MNRAS, 365, L11

Foreman-Mackey, D., Hogg, D. W., Lang, D., \& Goodman, J. 2013, PASP, 125 306

Gebhardt, K., Adams, J., Richstone, D., et al. 2011, ApJ, 729, 119

Georganopoulos, M., Meyer, E., \& Perlman, E. 2016, Galaxies, 4, 65

Giroletti, M., Hada, K., Giovannini, G., et al. 2012, A\&A, 538, L10

Hada, K., Kino, M., Doi, A., et al. 2016, ApJ, 817, 131

Harris, D. E., \& Krawczynski, H. 2002, ApJ, 565, 244

Harris, D. E., Biretta, J. A., Junor, W., et al. 2003, ApJ, 586, L41

Harris, D. E., Cheung, C. C., Biretta, J. A., et al. 2006, ApJ, 640, 211

Harris, D. E., Cheung, C. C., Stawarz, Ł., Biretta, J. A., \& Perlman, E. S. 2009, ApJ, 699, 305

Harris, D. E., Massaro, F., Cheung, C. C., et al. 2011, ApJ, 743, 177

Heinz, S., \& Begelman, M. C. 1997, ApJ, 490, 653

Jordán, A., Côté, P., Blakeslee, J. P., et al. 2005, ApJ, 634, 1002

Levinson, A., \& Globus, N. 2017, MNRAS, 465, 1608

Liu, W.-P., \& Shen, Z.-Q. 2007, ApJ, 668, L23

Liu, R.-Y., Rieger, F. M. \& Aharonian, F. A. 2017, ApJ, 842, 39

Macchetto, F., Marconi, A., Axon, D. J., et al. 1997, ApJ, 489, 579

Marshall, H. L., Miller, B. P., Davis, D. S., et al. 2002, ApJ, 564, 683

Massaro, E., Perri, M., Giommi, P., \& Nesci, R. 2004, A\&A, 413, 489

Meisenheimer, K., Roeser, H.-J., \& Schloetelburg, M. 1996, A\&A, 307, 61

Mertens, F., Lobanov, A. P., Walker, R. C., \& Hardee, P. E. 2016, A\&A, 595, A54

Meyer, E. T., Sparks, W. B., Biretta, J. A., et al. 2013, ApJ, 774, L21

Mościbrodzka, M., Falcke, H., \& Shiokawa, H. 2016, A\&A, 586, A38

Osone, S. 2017, ArXiv e-prints [arXiv: 1706.00683]

Ostrowski, M. 2000, MNRAS, 312, 579

Perlman, E. S., \& Wilson, A. S. 2005, ApJ, 627, 140

Perlman, E. S., Biretta, J. A., Zhou, F., Sparks, W. B., \& Macchetto, F. D. 1999 AJ, 117, 2185

Perlman, E. S., Biretta, J. A., Sparks, W. B., Macchetto, F. D., \& Leahy, J. P. 2001, ApJ, 551, 206

Perlman, E. S., Harris, D. E., Biretta, J. A., Sparks, W. B., \& Macchetto, F. D. 2003, ApJ, 599, L65

Perlman, E. S., Adams, S. C., Cara, M., et al. 2011, ApJ, 743, 119

Reynolds, C. S., Di Matteo, T., Fabian, A. C., Hwang, U., \& Canizares, C. R. 1996, MNRAS, 283, L111

Rieger, F. M., \& Aharonian, F. 2012, Mod. Phys. Lett. A, 27, 1230030

Rieger, F. M., Bosch-Ramon, V., \& Duffy, P. 2007, Ap\&SS, 309, 119

Russell, H. R., Fabian, A. C., McNamara, B. R., \& Broderick, A. E. 2015, MNRAS, 451, 588

Sahayanathan, S. 2008, MNRAS, 388, L49

Sparks, W. B., Biretta, J. A., \& Macchetto, F. 1996, ApJ, 473, 254 
A\&A 612, A106 (2018)

Tramacere, A., Massaro, F., \& Cavaliere, A. 2007, A\&A, 466, 521

Walsh, J. L., Barth, A. J., Ho, L. C., \& Sarzi, M. 2013, ApJ, 770, 86

Waters, C. Z., \& Zepf, S. E. 2005, ApJ, 624, 656

Weisskopf, M. C., Tananbaum, H. D., Van Speybroeck, L. P., \& O’Dell, S. L. 2000, in X-Ray Optics, Instruments, and Missions III, eds. J. E. Truemper, \& B. Aschenbach, Proc. SPIE, 4012, 2

Weisskopf, M. C., Brinkman, B., Canizares, C., et al. 2002, PASP, 114, 1
Wilson, A. S., \& Yang, Y. 2002, ApJ, 568, 133

Wilson, A. S., \& Yang, Y. 2004, ApJ, 610, 624

Yuan, F., \& Narayan, R. 2014, ARA\&A, 52, 529

Zabalza, V. 2015, in 34th International Cosmic Ray Conference (ICRC2015), Int. Cosmic Ray Conf., 34, 922

Zhang, J., Bai, J.-M., Chen, L., \& Yang, X. 2009, ApJ, 701, 423

Zhang, J., Bai, J. M., Chen, L., \& Liang, E. 2010, ApJ, 710, 1017 
Appendix A:

Table A.1. Chandra observations of M 87.

\begin{tabular}{|c|c|c|c|c|c|c|c|}
\hline ObsID & $\begin{array}{c}\text { Frame time } \\
(\mathrm{s})\end{array}$ & $\begin{array}{l}\text { Exp time } \\
(\mathrm{ks})\end{array}$ & $\begin{array}{c}\text { Start date } \\
\text { (YYY-MM-DD) }\end{array}$ & ObsID & $\begin{array}{c}\text { Frame time } \\
(\mathrm{s})\end{array}$ & $\begin{array}{l}\text { Exp time } \\
(\mathrm{ks})\end{array}$ & $\begin{array}{c}\text { Start date } \\
\text { (YYY-MM-DD) }\end{array}$ \\
\hline 1808 & 0.4 & 12.85 & $2000-07-30$ & 8578 & 0.4 & 4.71 & 2008-04-01 \\
\hline 3085 & 0.4 & 4.89 & $2002-01-16$ & 8579 & 0.4 & 4.71 & $2008-05-15$ \\
\hline 3084 & 0.4 & 4.65 & 2002-02-12 & 8580 & 0.4 & 4.7 & 2008-06-24 \\
\hline 3086 & 0.4 & 4.62 & 2002-03-30 & 8581 & 0.4 & 4.66 & 2008-08-07 \\
\hline 3087 & 0.4 & 4.97 & 2002-06-08 & 10282 & 0.4 & 4.7 & 2008-11-17 \\
\hline 3088 & 0.4 & 4.71 & $2002-07-24$ & 10283 & 0.4 & 4.7 & 2009-01-07 \\
\hline 3975 & 0.4 & 5.29 & $2002-11-17$ & 10284 & 0.4 & 4.7 & $2009-02-20$ \\
\hline 3976 & 0.4 & 4.79 & $2002-12-29$ & 10285 & 0.4 & 4.66 & 2009-04-01 \\
\hline 3977 & 0.4 & 5.28 & 2003-02-04 & 10286 & 0.4 & 4.68 & $2009-05-13$ \\
\hline 3978 & 0.4 & 4.85 & 2003-03-09 & 10287 & 0.4 & 4.7 & 2009-06-22 \\
\hline 3979 & 0.4 & 4.49 & 2003-04-14 & 10288 & 0.4 & 4.68 & $2009-12-15$ \\
\hline 3980 & 0.4 & 4.79 & 2003-05-18 & 11512 & 0.4 & 4.7 & 2010-04-11 \\
\hline 3981 & 0.4 & 4.68 & 2003-07-03 & 11513 & 0.4 & 4.7 & 2010-04-13 \\
\hline 3982 & 0.4 & 4.84 & 2003-08-08 & 11514 & 0.4 & 4.53 & 2010-04-15 \\
\hline 4917 & 0.4 & 5.03 & 2003-11-11 & 11515 & 0.4 & 4.7 & 2010-04-17 \\
\hline 4918 & 0.4 & 4.68 & 2003-12-29 & 11516 & 0.4 & 4.71 & 2010-04-20 \\
\hline 4919 & 0.4 & 4.7 & $2004-02-12$ & 11517 & 0.4 & 4.7 & $2010-05-05$ \\
\hline 4921 & 0.4 & 5.25 & 2004-05-13 & 11518 & 0.4 & 4.4 & 2010-05-09 \\
\hline 4922 & 0.4 & 4.54 & 2004-06-23 & 11519 & 0.4 & 4.71 & 2010-05-11 \\
\hline 4923 & 0.4 & 4.63 & $2004-08-05$ & 11520 & 0.4 & 4.6 & 2010-05-14 \\
\hline 5737 & 0.4 & 4.21 & $2004-11-26$ & 13964 & 0.4 & 4.54 & 2011-12-04 \\
\hline 5738 & 0.4 & 4.67 & $2005-01-24$ & 13965 & 0.4 & 4.6 & $2012-02-25$ \\
\hline 5739 & 0.4 & 5.15 & 2005-02-14 & 14974 & 0.4 & 4.6 & $2012-12-12$ \\
\hline 5740 & 0.4 & 4.7 & 2005-04-22 & 14973 & 0.4 & 4.4 & 2013-03-12 \\
\hline 5744 & 0.4 & 4.7 & $2005-04-28$ & 16042 & 0.4 & 4.62 & $2013-12-26$ \\
\hline 5745 & 0.4 & 4.7 & $2005-05-04$ & 16043 & 0.4 & 4.6 & 2014-04-02 \\
\hline 5746 & 0.4 & 5.14 & 2005-05-13 & 17056 & 0.4 & 4.6 & 2014-12-17 \\
\hline 5747 & 0.4 & 4.7 & $2005-05-22$ & 17057 & 0.4 & 4.6 & 2015-03-19 \\
\hline 5748 & 0.4 & 4.7 & $2005-05-30$ & 18233 & 0.4 & 37.25 & $2016-02-23$ \\
\hline 5741 & 0.4 & 4.7 & 2005-06-03 & 18781 & 0.4 & 39.51 & $2016-02-24$ \\
\hline 5742 & 0.4 & 4.7 & 2005-06-21 & 18782 & 0.4 & 34.07 & $2016-02-26$ \\
\hline 5743 & 0.4 & 4.67 & 2005-08-06 & 18809 & 0.4 & 4.52 & 2016-03-12 \\
\hline 6299 & 0.4 & 4.65 & $2005-11-29$ & 18810 & 0.4 & 4.6 & 2016-03-13 \\
\hline 6300 & 0.4 & 4.66 & 2006-01-05 & 18811 & 0.4 & 4.6 & 2016-03-14 \\
\hline 6301 & 0.4 & 4.34 & 2006-02-19 & 18812 & 0.4 & 4.4 & 2016-03-16 \\
\hline 6302 & 0.4 & 4.7 & 2006-03-30 & 18813 & 0.4 & 4.6 & 2016-03-17 \\
\hline 6303 & 0.4 & 4.7 & 2006-05-21 & 18783 & 0.4 & 36.11 & 2016-04-20 \\
\hline 6304 & 0.4 & 4.68 & $2006-06-28$ & 18232 & 0.4 & 18.2 & 2016-04-27 \\
\hline 6305 & 0.4 & 4.65 & 2006-08-02 & 18836 & 0.4 & 38.91 & 2016-04-28 \\
\hline 7348 & 0.4 & 4.54 & 2006-11-13 & 18837 & 0.4 & 13.67 & 2016-04-30 \\
\hline 7349 & 0.4 & 4.68 & 2007-01-04 & 18838 & 0.4 & 56.29 & $2016-05-28$ \\
\hline 7350 & 0.4 & 4.66 & $2007-02-13$ & 18856 & 0.4 & 25.46 & 2016-06-12 \\
\hline 8510 & 0.4 & 4.7 & $2007-02-15$ & 517 & 3.2 & 6.99 & $2000-04-15$ \\
\hline 8511 & 0.4 & 4.7 & $2007-02-18$ & 241 & 3.2 & 38.04 & $2000-07-17$ \\
\hline 8512 & 0.4 & 4.7 & $2007-02-21$ & 352 & 3.2 & 37.68 & $2000-07-29$ \\
\hline 8513 & 0.4 & 4.7 & $2007-02-24$ & 3717 & 3.2 & 20.56 & $2002-07-05$ \\
\hline 8514 & 0.4 & 4.47 & 2007-03-12 & 2707 & 3.2 & 98.66 & $2002-07-06$ \\
\hline 8515 & 0.4 & 4.7 & 2007-03-14 & 6186 & 3.2 & 51.55 & 2005-01-31 \\
\hline 8516 & 0.4 & 4.68 & 2007-03-19 & 7212 & 3.1 & 65.25 & 2005-11-14 \\
\hline 8517 & 0.4 & 4.67 & $2007-03-22$ & 7210 & 3.1 & 30.71 & 2005-11-16 \\
\hline 7351 & 0.4 & 4.68 & $2007-03-24$ & 7211 & 3.1 & 16.62 & $2005-11-16$ \\
\hline 7352 & 0.4 & 4.59 & $2007-05-15$ & 5828 & 3.1 & 32.99 & 2005-11-17 \\
\hline 7353 & 0.4 & 4.54 & $2007-06-25$ & 6186 & 3.1 & 51.55 & 2005-01-31 \\
\hline 7354 & 0.4 & 4.71 & $2007-07-31$ & 5827 & 3.1 & 156.2 & $2005-05-05$ \\
\hline 8575 & 0.4 & 4.68 & $2007-11-25$ & 5826 & 3.1 & 126.76 & $2005-03-03$ \\
\hline 8576 & 0.4 & 4.69 & 2008-01-04 & & & & \\
\hline 8577 & 0.4 & 4.66 & $2008-02-16$ & & & & \\
\hline
\end{tabular}

